\title{
New Early Warning Indicator Based on Vehicle Load and Difference Cur- vature for Simply-Supported Beam Bridges
}

\author{
Zhongjun $\mathrm{Ma}^{1,2 *}$, Yuzhou Sima ${ }^{2}$ and Guochao Chen ${ }^{2}$ \\ ${ }^{I}$ Wuhan University of Technology, Wuhan 430070, P.R. China \\ ${ }^{2}$ Nanyang Institute of Technology, Nanyang 473004, P.R. China
}

\begin{abstract}
A new indicator called Vehicle Load Difference Curvature Indicator (VLDCI) was proposed in this paper to further study the application of difference curvature to early warning of simply supported beams. Values of VLDCI can be obtained based on the measured vertical displacement data under a novel vehicle load mode (working condition of concentrated load), which can be obtained by apply vehicle loading twice. The graph of VLDCI can indicate the damage information. Case study shows that VLDCI method can detect the occurrence and position of damage effectively and then the early warning of beam status can be realized. Moreover, the calculation process of this new indicator is simple while VLDCI values of the intact beams are not required.
\end{abstract}

Keywords: Curvature, damage, early warning, simply supported beam.

\section{INTRODUCTION}

Simply supported beams find a widespread application in infrastructure construction and industrial equipment. To ensure normal function and avoid accidents, it is necessary to perform early warning on the operation status of the simply supported beams. Once damage appears, maintenance and disposition is required. For different simply supported beams, different modes of damage can be observed. For example, steel corrosion, crack, concrete cracking, caving, and so on. These damages, however, can be uniformly characterized by the change of the local bending stiffness in theory [1].

At present, the investigations on the damage identification is thriving, and several damage identification indexes have been established. Based on the variation of these indexes, the damage of structure can be identified [2-4]. Among these indexes, the index based on the curvature concept is very remarkable. It mainly includes two categories: the methods based on the dynamic effect and static effect, respectively. The former specifically refers to the index based on the curvature modes. The basic idea is to find the damage by comparing the modal curvature graph of the structures before and after damage [5-8]. The latter refers to the index based on the differential curvature. This index is obtained by processing the static displacement data of the structure through the central difference approximation method. It can also indicate the damage characteristics.

Theoretically, both of the methods are effective. However, both suffers to some obstacles in practical applications:
(1) For the curvature mode based index, it is very difficult to obtain the ideal curvature modal data due to the limitation of the number and accuracy of acceleration sensors. Consequently, this damage identification index is not obviously sensitive to the damage, and misdiagnosis will be arrived occasionally. Furthermore, it is relatively complex to obtain the curvature mode from the original measurements, which indicates that this method is not conducive to the efficiency of early warning; (2) For the differential curvature based index, investigations on the appropriate static loading methods is on the way. On one hand, the loading devices in the laboratory are difficult to be used in practical field, on the other hand there are many restrictions in the range, beam scale and support conditions.

Therefore, it is necessary to further study the damage detection of a simply supported beam based on the curvature concept. In view of loading methods, the most appropriate method is to apply the vehicle load. For example, it is convenient to directly drive a truck on the large beam structure; while special small vehicles can be applied for small beam structures. It is not only convenient to adjust the load value, but also convenient for moving, loading, and unloading. However, few reports can be found to combine the vehicle loading with the static differential curvature index at present.

The present paper attempts to obtain the damage information of the simply supported beams using the differential curvature data under specific vehicle, and then realizes the damage state warning. The computing process of the present new method is simple while it is easy to achieve the early warning. Meanwhile, it is difficult to apply the same loading to the intact structure for comparison. Consequently, the newly developed method is able to identify the damage in cases where only the measurement data of the damaged structure are available. 


\section{METHOD ESTABLISHMENT}

According to the central difference approximation method, the difference curvature at a certain point, $i$, of the beam can be calculated by:

$$
\frac{1}{\rho_{i}}=\frac{M_{i}}{B_{i}}=\frac{y_{i+1}-2 y_{i}+y_{i-1}}{\Delta^{2}}
$$

where $\Delta$ is the measuring point distance; $\mathrm{Mi}, \mathrm{Bi}$ and yi refers to the bending moment, bending stiffness and static vertical displacement at the point $\mathrm{i}$, respectively; yi+1 and yi-1 refers to the static vertical displacements at two points which are at an axial distance of $\Delta$ from the point $i$, respectively.

In the case of a simply supported beam with a span of $\mathrm{L}$, it is firstly divided into segments with a distance of $\Delta$. The number of virtual segments should be an even number. The boundary points of each virtual segments are considered as the measurement points of the vertical displacement (as shown in Fig. (1). Suppose that damage occurs somewhere between the points $i$ and $i+1$. The damage area has a width of $y$, and is at a distance of $x$ from the left end of the beam. In the damage region, local bending stiffness changes from $\mathrm{B}$ to $\mathrm{zB}$.

The load is applied by using the vehicle. The load are applied twice using two different vehicles. The specific methods are as following:

(1) Vehicle 1 loading; The front wheel of the vehicle1 is parked in the middle of the span. The loads provided by the front and rear axles are approximately considered as concentrated loads, named as F1 and F2 (as shown in Fig. (2). The vertical displacements at each measuring points are recorded. Subsequently, the vehicle 1 leaves the beam.

(2) Vehicle 2 loading: The front and rear wheels of vehicle 2 are parked at the same locations as the vehicle 1 . However, the type of the vehicle 2 is different from that of the vehicle 1 . The concentrated load provided by the rear axle should also be F1 through adjusting the weight ratio of the front and rear axles. Meanwhile, the concentrated load provided by the front axle is F3 (F3<F2), as shown in Fig. (3). The vertical displacements at each measuring points are recorded. Subsequently, the vehicle 2 leaves the beam.

Based on the elastic theory, the difference of the two series displacement measurements for each measuring point can be considered as the displacement generated by the concentrated load (F2-F3) in the midspan. Because most of the simply supported beams are not deep beam, the shear deformation can be ignored. Assuming that the measuring point i1 is $m$ apart from the left end of the beam. According to the virtual work principle, we can obtain the vertical displacement at each measuring point generated by the midspan load (F2-F3):

The vertical displacement at measuring point i-1, i.e., yi1 , is calculated as

$$
y_{i-1}=\frac{-\mathrm{c} F_{2}-F_{3} \quad m\left[24 x y(x+y)(1-z)-3 l^{3} z-12 l y^{2}(1-z) \quad 8 y^{3}(1-z)-24 l x y(1-z)+4 l m^{2} z\right]}{48 l z B}
$$

The vertical displacement at measuring point $i$, i.e., yi, is calculated as

$$
\begin{aligned}
& y_{i}=\frac{-F_{2}-F_{3}(\Delta+m)\left[24 x y(x+y)-3 l^{3} z-12 l y^{2}(1-z)+8 y^{3}(1-z)\right.}{48 l z B} \\
& \frac{\left.-24 \operatorname{lx} y(1-z)+4 l z\left(\Delta^{2}+m^{2}\right)-24 x y z(x+y)+8 \Delta l m z\right]}{48 l z B}
\end{aligned}
$$

The vertical displacement at measuring point

$\mathrm{i}+1$, i.e., $\mathrm{yi}+1$, is calculated as:

$$
\begin{aligned}
y_{i+1} & =\frac{-c F_{2}-F_{3}\left[24 x y(x+y)(2 \Delta+m-l-2 z+z l-z m)+8 y^{3} z(l-m)\right.}{48 l z B} \\
& +\frac{\left.24 \Delta l m^{2} z(m+2 \Delta)+16 \Delta y^{3}(1-z)-8 y^{3}(l-m)-6 \Delta l^{3} z+32 \Delta^{3} l z+4 l m^{3} z-3 l^{3} m z\right]}{48 l z B}
\end{aligned}
$$

Subsequently, the differential curvature at measuring point $i$ is calculated as:

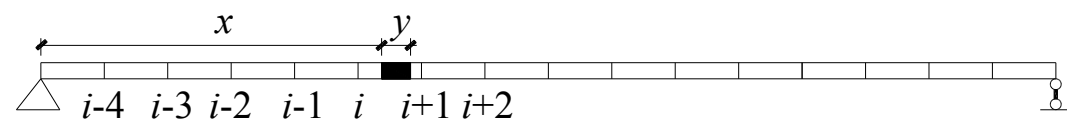

Fig. (1). Model of damaged simply supported beam.

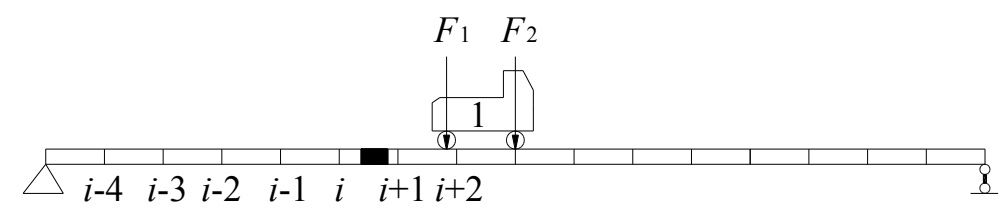

Fig. (2). Sketch of Vehicle 1 loading.

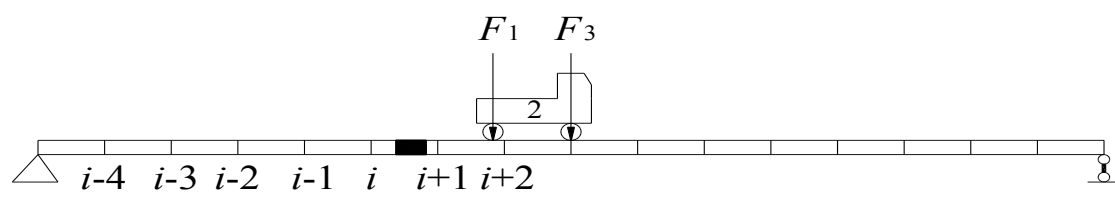

Fig. (3). Sketch of Vehicle 2 loading. 


$$
\begin{aligned}
& \frac{1}{\rho_{i}}=\frac{-\mathrm{c} F_{2}-F_{3}\left[6 \Delta y^{2}(1-z)+6 \Delta^{2} z(\Delta+m)\right.}{12 z \Delta^{2} B} \\
& \frac{+3 m y^{2}(1-z)-6 x y(x+y)(1-z)}{12 z \Delta^{2} B} \\
& \frac{-c F_{2}-F_{3}\left[-2 y^{3}(1-z)+12 \Delta x y(1-z)+6 m x y(1-z)\right]}{12 z \Delta^{2} B}
\end{aligned}
$$

It can be seen the differential curvature at measuring point $\mathrm{i}$ includes the damage information $(\mathrm{x}, \mathrm{y}, \mathrm{z})$.

Similarly, the differential curvature at measuring point $\mathrm{i}+1$ is calculated as:

$$
\begin{aligned}
& \frac{1}{\rho_{i+1}}=\frac{-\mathrm{c} F_{2}-F_{3}\left[24 \Delta y(1-z)\left(l y-x y-x^{2}+2 l x\right)\right.}{12 z \Delta^{2} B} \\
& \frac{-8 y^{3}(1-z)(\Delta+m)+3 l^{3} z(\Delta+m)+68 \Delta^{3} l z}{12 z \Delta^{2} B} \\
& \frac{-c F_{2}-F_{3}\left[12 \operatorname{lm}\left(y^{2}-\Delta m z-y^{2} z\right)\right.}{12 z \Delta^{2} B} \\
& \frac{\left.-4 m^{3} z-24 m x y(x+y-y z-x z-l-l z)+36 \Delta^{2} l m z\right]}{12 z \Delta^{2} B}
\end{aligned}
$$

The differential curvature at measuring point $i+2$ is calculated as:

$$
\frac{1}{\rho_{i+2}}=\frac{-c F_{2}-F_{3}(m+3 \Delta)}{2 B}
$$

The differential curvature at measuring point $i+3$ is calculated as:

$$
\frac{1}{\rho_{i+3}}=\frac{-c F_{2}-F_{3}(m+4 \Delta)}{2 B}
$$

The differential curvature at measuring point $i+4$ is calculated as:

$$
\frac{1}{\rho_{i+4}}=\frac{-c F_{2}-F_{3}(m+5 \Delta)}{2 B}
$$

The differential curvature at measuring point $i+5$ is calculated as:

$$
\frac{1}{\rho_{i+5}}=\frac{-c F_{2}-F_{3}(m+6 \Delta)}{2 B}
$$

Consequently, we obtain:

$$
\frac{1}{\rho_{i+3}}-\frac{1}{\rho_{i+2}}=\frac{1}{\rho_{i+4}}-\frac{1}{\rho_{i+3}}=\bullet \bullet \bullet \bullet=\frac{-c F_{2}-F_{3} \Delta}{2 B}
$$

For the left side, the differential curvature at measuring point $i-1$ is calculated as:

$$
\frac{1}{\rho_{i-1}}=\frac{-\mathrm{c} F_{2}-F_{3} \quad m}{2 B}
$$

The differential curvature at measuring point i-2 is calculated as:

$$
\frac{1}{\rho_{i-2}}=\frac{-\mathrm{c} F_{2}-F_{3}(m-\Delta)}{2 B}
$$

The differential curvature at measuring point i-3 is calculated as:

$$
\frac{1}{\rho_{i-3}}=\frac{-\mathrm{c} F_{2}-F_{3}(m-2 \Delta)}{2 B}
$$

The differential curvature at measuring point i-4 is calculated as:

$$
\frac{1}{\rho_{i-4}}=\frac{-\mathrm{c} F_{2}-F_{3}(m-3 \Delta)}{2 B}
$$

The differential curvature at measuring point i-5 is calculated as:

$$
\frac{1}{\rho_{i-5}}=\frac{-c F_{2}-F_{3}(m-4 \Delta)}{2 B}
$$

Consequently, we obtain:

$$
\frac{1}{\rho_{i-1}}-\frac{1}{\rho_{i-2}}=\frac{1}{\rho_{i-2}}-\frac{1}{\rho_{i-3}}=\cdots \bullet \bullet \bullet=\frac{-c F_{2}-F_{3} \Delta}{2 B}
$$

Based on above results, an index, V, i.e., Vehicle Load Difference Curvature Indicator (VLDCI), can be defined as:

$V_{i}=\frac{1}{\rho_{i}}-\frac{1}{\rho_{i-1}}$

Consequently, we obtain

$V_{i-1}=V_{i-2}=\cdots=\frac{-\mathrm{c} F_{2}-F_{3} \Delta}{2 B}$
$V_{i+3}=V_{i+4}=\cdots=\frac{-\mathrm{c} F_{2}-F_{3} \Delta}{2 B}$

For the measuring point at the left end of the damage region, i:

$$
\begin{aligned}
& V_{i}=\frac{1}{\rho_{i}}-\frac{1}{\rho_{i-1}}=\frac{-c F_{2}-F_{3}\left[6 \Delta y^{2}(1-z)+6 \Delta^{3} z\right.}{12 z \Delta^{2} B} \\
& \frac{\left.+3 m y^{2}(1-z)-2 y^{3}(1-z)\right]}{12 z \Delta^{2} B} \\
& \frac{-c F_{2}-F_{3}[-6 x y(x+y)(1-z)}{12 z \Delta^{2} B} \\
& \frac{+12 \Delta x y(1-z)+6 m x y(1-z)]}{12 z \Delta^{2} B}
\end{aligned}
$$

Both $\mathrm{Vi}+2$ and $\mathrm{Vi}+3$ will not be in accord with the simple law of Eq. (2). It can be seen that when the damage occurs between the measuring points $i$ and $i+1$, the VLDCI values at three points, namely $\mathrm{i}, \mathrm{i}+1, \mathrm{i}+2$, will change. Consequently, this index has the ability to achieve the early warning. The specific implementation method is as follows: for a simply supported beam, two vehicles are loaded at the specified location mentioned above. The vertical displacements at each measuring point are recorded under two loading condi- 
tions. The corresponding subtraction of two groups of data are conducted to obtain the VLDCI value at each measuring point. Subsequently, the X-Y curve is plotted, and the catastrophe location will imply the damage position. Consequently, the early warning message of the beam state can be obtained. One can perform specific inspection on the marked position, confirm the damage situation, and make treatments in time for guaranteeing the safety.

\section{CASE}

The investigated simply supported beam was fabricated by Q345 steel and has a span of $20 \mathrm{~m}$. The box section is $5 \mathrm{~m}$ in width, and $2 \mathrm{~m}$ in height. Its wall thickness is $20 \mathrm{~mm}$. The finite element model is set up by MIDAS/CIVIL, and the numerical simulation is performed using the spatial beam element.

\section{(1) Damage setting}

The damage occurs at $\mathrm{x}=6 \mathrm{~m}$, and $\mathrm{y}=0.2 \mathrm{~m}$. The sectional bending stiffness in the damage region decreases by $5 \%$.

\section{(2) Loading and results}

The spacing of vertical displacement measuring points is $\Delta=0.5 \mathrm{~m}$. The loading is firstly performed using the vehicle 1 whose front axle is parked in the middle of the span. The vehicle 1 is $1000 \mathrm{kN}$ in weight, and its rear axle introduces a load of $900 \mathrm{kN}$. Subsequently, the vehicle 2 is parked in the middle of the span as vehicle 1. Its front axle introduce a load of $200 \mathrm{kN}$ while the rear axle introduce $900 \mathrm{kN}$. Consequently, it can be considered that an equivalent concentrated load $(\mathrm{F} 2-\mathrm{F} 3=800 \mathrm{kN})$ is applied in the middle of the span. Based on the Eq. (1) and the displacement data calculated by MIDAS, we obtain the $\mathrm{V}$ as a function of the axial location, partially shown in Fig. (4).

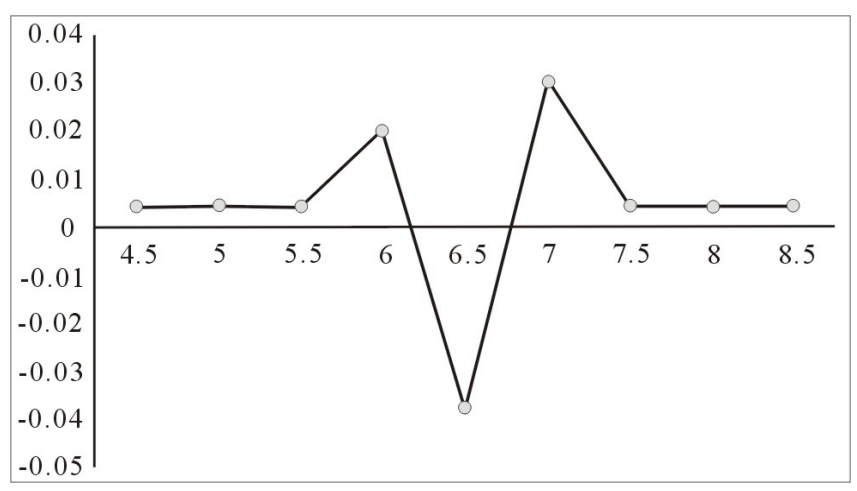

Fig. (4). Graph of V values.

The horizontal coordinate, $\mathrm{x} 1$, originates from the left end of the beam, and the unit is $\mathrm{m}$; the vertical coordinate refers to $\mathrm{V}$, and the unit is $1 /(103 \mathrm{~m})$. It can be seen that the mutation point appears at $x^{\prime}=6 \mathrm{~m}$, illustrating that the damage occurs in the interval $[6 \mathrm{~m}, 6.5 \mathrm{~m}]$. This result is consistent with the damage setting. Present case demonstrates that the VLDCI index can effectively locate the damage and provide early warning information.

\section{SUGGESTIONS ON THE CALCULATION STABIL- ITY IMPROVEMENT}

The mutation amplitude of the VLDCI value caused by the local damage between the measuring points $i$ and $i+1$ is calculated as:

$$
\begin{aligned}
& \left|V_{i}-V_{i-1}\right|=\left|\frac{1}{\rho_{i}}-\frac{2}{\rho_{i-1}}+\frac{1}{\rho_{i-2}}\right| \\
& =\frac{\mathrm{c} F_{2}-F_{3}\left[6 \Delta y^{2}(1-z)+3 m y^{2}(1-z)-6 x y(x+y)(1-z)\right]}{12 z \Delta^{2} B} \\
& \frac{+\mathrm{c} F_{2}-F_{3}\left[6 \Delta^{2} m z-2 y^{3}(1-z)+12 \Delta x y(1-z)+6 m x y(1-z)\right]}{12 z \Delta^{2} B}
\end{aligned}
$$

To improve the stability of the new method, a larger mutation amplitude is required to illustrate the damage characteristics more clearly. According to Eq. (3), two measures can be adopted as follows:

(1) In premise of not causing further damage, the (F2-F3) value, included in the numerator, should be as large as possible;

(2) The $\Delta$, included in the denominator, should be as small as possible, i.e., the measuring points should be arranged as densely as possible;

In addition, it is necessary to improve the accuracy of the displacement data.

\section{CONCLUSION}

Early warning of a damaged simply supported beam is still under investigation. The main obstacle is damage identification. The damage indicator based on curvature possesses many obvious difficulties in practical application although many advantages can be proved. In the present paper, a new damage detection indicator, i.e., Vehicle Load Difference Curvature Indicator (VLDCI), is developed by combining the vehicle loading and the static differential curvature method. The corresponding damage detection method is established. The main characteristics of this new method include: (1) According to the beam situation, it is convenient to use different types of vehicle for loading; (2) The calculation process is simple and clear; (3) If a damage appears on the beam, the damage location can be indicated; (4) Only the loading test on the damaged beam is required. Case study shows that the VLDCI can detect the damage location of a simply supported beam and further realize early warning efficiently and easily.

\section{CONFLICT OF INTEREST}

The author confirms that this article content has no conflict of interest.

\section{ACKNOWLEDGEMENT}

Declared none. 


\section{REFERENCES}

[1] R. O. Curadelli, J. D. Riera, D. Ambrosini, and M. G. Amani, "Damage detection by means of structural damping identification", Engineering Structures, vol. 30, no. 12, pp. 3497-3504, 2008.

[2] E. J. Yu and L. Chung, "Seismic damage detection of a reinforced concrete structure by finite element model updating", Smart Structures and Systems, vol. 9, no. 3, pp. 253-271, 2012.

[3] J. P. Tang, D. J. Chiou, C. W. Chen, W. L. Chiang, W. K. Hsu, C. Y. Chen, and T. Y. Liu, "A case study of damage detection in benchmark buildings using a Hilbert-Huang Transform-based method", Journal of Vibration and Control, vol. 17, no. 4, pp. 623636, 2011

[4] Z. L. Liu, "Fractal theory and application in city size distribution", Information Technology Journals, vol. 12, no. 17, pp. 4158-4162, 2013. doi:10.3923/itj.2013.4158.4162.
[5] A. Tomaszewska, "Influence of statistical errors on damage detection based on structural flexibility and mode shape curvature", Computers and Structures, vol. 88, no. 3-4, pp. 154-164, 2010.

[6] M. Chandrashekhar and R. Ganguli, "Damage assessment of structures with uncertainty by using mode-shape curvatures and fuzzy logic", Journal of Sound and Vibration, vol. 326, no. 3-5, pp. 939957, 2009.

[7] C. S. Hamey, W. Lestari, P. Z. Qiao, and G. B. Song, "Experimental damage identification of carbon/epoxy composite beams using curvature mode shapes", Structural Health Monitoring, vol. 3, no. 4, pp. 333-353, 2004.

[8] A. Dutta and S. Talukdar, "Damage detection in bridges using accurate modal parameters", Finite Element Analysis and Design, vol. 40 , no. 3 , pp. 287-304, 2004.

Received: September 16, 2014

Revised: December 23, 2014

Accepted: December 31, 2014

(C) Ma et al.; Licensee Bentham Open.

This is an open access article licensed under the terms of the (https://creativecommons.org/licenses/by/4.0/legalcode), which permits unrestricted, non-commercial use, distribution and reproduction in any medium, provided the work is properly cited. 